\title{
Viscosity Measurement Techniques in dissipative Particle Dynamics
}

\author{
Arman Boromand ${ }^{1}$, Safa Jamali ${ }^{1}$, Joao M. Maia* \\ 1Department of Macromolecular Science and Engineering, Case Western Reserve University, Cleveland, \\ OH 44106, USA \\ * - Author to whom correspondence should be addressed. Email: joao.maia@case.edu; T: +1-216-5432454 \\ F: $+1-216-3684202$
}

$\mathrm{AB}$ and $\mathrm{SJ}$ contributed equally in preparing the manuscript and performing the research

\section{Abstract:}

In this study two main groups of viscosity measurement techniques are used to measure the viscosity of a simple fluid using Dissipative Particle Dynamics, DPD. In the first method, a microscopic definition of the pressure tensor is used in equilibrium and out of equilibrium to measure the zero-shear viscosity and shear viscosity, respectively. In the second method, a periodic Poiseuille flow and start-up transient shear flow is used and the shear viscosity is obtained from the velocity profiles by a numerical fitting procedure. Using the standard Lees-Edward boundary condition for DPD will result in incorrect velocity profiles at high values of the dissipative parameter. Although this issue was partially addressed in [Chatterjee, A., Molecular Simulation, 2007. 33(15): p. 1233-1236], in this work we present further modifications (Lagrangian approach) to the original LE boundary condition (Eulerian approach) that will fix the deviation from the desired shear rate at high values of the dissipative parameter and decrease the noise to signal ratios in stress measurement while increases the accessible low shear rate window. Also, the thermostat effect of the dissipative and random forces is coupled to the dynamic response of the system and affects the transport properties like the viscosity and diffusion coefficient. We investigated thoroughly the dependency of viscosity measured by both Eulerian and Lagrangian methodologies, as well as numerical fitting procedures and found that all the methods are in quantitative agreement. 


\section{Introduction}

Despite the tremendous growth of the computational power and capabilities over the past decades, atomistic models are still limited to the very short times and length scales. To overcome the deficiency of the atomistic models from the computational perspective, there has been a great interest in coarse-grain models, since they provide the chance to reach time and length scales much larger by comparison with atomistic models, and with lower computational cost. Dissipative Particle Dynamics (DPD) was introduced to the scientific community in 1992 [1] as a coarse-grained numerical model based on Molecular Dynamics (MD) method. The coarse-grained nature of DPD enables much larger time and length scales compared to MD simulations to be achieved, thus great enthusiasm was shown towards using DPD since its early introduction. Although numerous contributions have used DPD as the main subject of the study, many aspects of DPD method are rather unexplored. On one hand, simplified formulation and use of soft potentials with only a few controlling parameter makes the method a perfect candidate for use in simulation of a wide range of different phenomena. On the other hand, the coarsegrained nature of the potentials introduces numerical artifacts that prevent this formalism from being applied to more complex systems and applications, such as polymer melts, blends and composites.

A DPD particle interacts with its neighbors through three effective forces (to be explained in detail in section II) called conservative, random and dissipative, with each force being measured via a controlling parameter. In other words, these three parameters define the system being modeled. Interestingly, there are only two expressions at hand to set these parameters: (I) the conservative force controlling parameter can be decided using the well-known Groot-Warren [2] formulation based on compressibility of a component, and (II) the dissipative and random parameters together define the dimensionless temperature in the system and thus can be chosen by solving the fluctuation-dissipation

relationship [3]. Despite the crucial role of dissipative and random parameters in defining the dynamics of a DPD system, they can take any arbitrary value as long as the dissipation-fluctuation relationship is satisfied. Due to the lack of an expression to provide unique values for these parameters, and perhaps due to the initial introduction in the method of values of 3.0 and 4.5 for random and dissipative parameters respectively, the majority of the works reported in the literature have used this same parameter set.

Regardless of the parameter choices and the material subject to study, one can categorize any simulation into two different conditions:

i) Equilibrium simulations, where material properties are monitored at rest (and by definition replicates the behavior of the fluid in the linear regime); 
ii) Non-equilibrium conditions, in which DPD particles are subject to external fields and ratedependent properties of a fluid can be reproduced.

The former involves simple periodic boundary conditions and yields consistent and thermally stable results, even though they sometimes suffer from statistical issues due to very poor signal-to-noise ratio, especially in the measurement of stress and thermodynamic quantities. On the other hand, nonequilibrium simulations in general lead to more stable and consistent calculations, but suffer from two sources of artifacts. Firstly, the traditional Lees-Edwards boundary condition is not capable of maintaining stable profiles at the boundaries in velocity direction, and therefore additional modifications are required. Secondly, since the dissipative and random forces together serve as a built-in thermostat for DPD and contribute to dynamics of the system at the same time, out of equilibrium simulations can affect the temperature controlling effect of the dissipative force and hence the thermal stability of the calculations.

Different boundary conditions (BCs) including wall-bounded and unbounded, periodic Poiseulle flow (PPF), use of ghost particles and bounce back, etc. have been proposed as modifications for nonequilibrium DPD simulations. For example, Pivkin et al. $[4,5]$ proposed a wall-bounded BC which induces the shear profile in the DPD domain by moving the solid wall particles. Backer and coworkers [6] proposed a periodic Poiseulle flow BC that results in stable velocity profiles and does not involve steady shear flow issues. However, for many applications/systems the method of choice is still steady shear since it imposes a constant shear rate along the calculation domain and using PPF can lead to secondary flows e.g. migration of internal constituents in the case of multiphasic systems or even polymer solutions or melts. Chatterjee [7], in an effort to modify the Lees-Edwards boundary conditions, introduced ghost particles in limiting boundaries in the velocity direction and obtained stable velocity profiles over a wide range of strains. Nevertheless, using such particles makes reliability of the ensemble averages and periodicity of the system questionable.

Different additional thermostats and built-in alternatives to DPD thermostat have been proposed in order to compensate for the shortcoming of the dissipative-random ensemble in temperature stabilization. Whittle and Travis [8] studied different external (MD-based) thermostats and their performance on controlling the temperature of a colloidal suspension under shear. Khani et al. [9] studied the temperature controlling effect of so-called Lowe-Anderson thermostat as an alternative to traditional DPD forces in polymer melts and found better stability of both temperature and dynamics using this method when compared to the simple dissipative-random ensemble. However, they have shortcomings at predicting the transport properties. 
Perhaps one of the most interesting quantities of a DPD fluid, one that reveals information about the dynamics of a system, is its viscosity. Many efforts have been made to study the rheology of a DPD fluid at different conditions. Marsh et. al. [10] derived an expression for the effect of dissipative parameter on the viscosity of a DPD fluid when there is no conservative force applied on the DPD particle. Different groups have studied rheological behavior of suspensions using both traditional and modified DPD [11-15]. Polymer melts and solutions, as well as multiphase systems have also been studied with special emphasis on their rheological behavior using DPD [9, 16, 17]. However, one can conclude from a careful review of the prior publications that, even for the simplest system (water, first studied by Groot and Warren [2]), values reported for the viscosity vary from one report to another, depending on the type of flow and method used for calculation of viscosity, boundary conditions and the choice of force parameters [17-20]. In particular, different viscosity values from Green-Kubo [21] expression for stress autocorrelation function (zero-shear viscosity), stress tensor for steady shear simulations, Poiseuille flow and transient start-up shear flow have been reported. Furthermore, theoretical predictions for temperature and dissipative parameter dependence of the viscosity are contradictory within the existing literature.

In this work we present a comprehensive study on different viscosity measurement methods and the effect of simulation parameters/conditions on rheological properties obtained by DPD simulations and provide guidance on the suitability of each method to researchers looking at using DPD to simulate the non-linear rheological behavior of complex fluids. To do so, in Section II a brief background on DPD formulation and algorithm is given, followed by a Section (III) dedicated to boundary conditions. There we introduce Lagrangian approach as a method to impose shear and compare out results to the LeesEdwards boundary conditions that is called Eulerian in the context of this paper. In Sections IV and V different DPD rheometry techniques are discussed: Section IV is dedicated to zero-shear viscosity measurements through the Green-Kubo autocorrelation function, and steady shear viscosity measurement using micro-stress tensor, while Section V explains the numerical fitting procedures required for viscosity calculations through Poiseuille flow and transient shear viscosity. In each section, the effect of different simulation parameters with emphasis on dissipative parameter is studied in order to give insight about the physical significance of this parameter. 


\section{Simulation background}

The equation of motion for a DPD particle is written based on the sum of three pairwise interaction forces between any $i j$ particle pair. For a single DPD particle $i$, Newton second law of motion can be written as:

$$
m_{i} \frac{d \mathbf{v}_{i}}{d t}=\sum \mathbf{F}_{i j}^{C}+\mathbf{F}_{i j}{ }^{D}+\mathbf{F}_{i j}{ }^{R} ; \frac{d \mathbf{r}_{i}}{d t}=\mathbf{v}_{i}
$$

The sum runs over all the $\mathrm{j}$ particles that are in a certain distance, called cut-off distance, from the $i^{\text {th }}$ particle and it is set as $r_{c}=1.0$ in all the simulations presented in this paper.

$\mathbf{F}_{i j}^{C}$, the conservative part of the total force, is a soft linearly decreasing repulsion force which represents the extent of interaction between different components and is given by

$$
\mathbf{F}_{i j}^{C}=a_{i j} w_{i j}^{C}\left(\mathrm{r}_{i j}\right) \mathbf{e}_{i j}
$$

Where $\mathrm{w}_{\mathrm{ij}}^{\mathrm{C}}$ is the linearly decreasing function of the distance between any pair of particles $\mathbf{r}_{i j}=\mathbf{r}_{i}-\mathbf{r}_{j} ; r_{i j}=\left|\mathbf{r}_{i j}\right| ; \mathbf{e}_{i j}=\frac{\mathbf{r}_{i j}}{r_{i j}}$, which goes to zero at the cut-off distance. The magnitude of the repulsive force is set by a parameter $a_{i j}$, which is the maximum repulsion between particle $\mathrm{i}$ and $\mathrm{j}$. Groot and Warren [2] proposed a formalism that utilizes the compressibility of a component $\left(\kappa^{-1}\right)$, in addition to temperature $\left(k_{B} T\right)$ and density $(\rho)$ used in the simulation to calculate the conservative force parameter which is given by

$$
a_{i j} \approx k_{B} T \frac{\kappa^{-1}-1}{0.2 \rho}
$$

In (eq.1), $\mathbf{F}_{i j}^{R}$ the random force is a stochastic force that introduces thermal fluctuations of DPD particles. $\mathbf{F}_{i j}^{D}$, the dissipative force, on the other hand, acts against relative motion of a particle pair and tries to reduce the kinetic energy of the system and describes the viscous resistance of the fluid. These forces are given by:

$$
\mathbf{F}_{i j}^{R}=\sigma_{i j} w_{i j}^{R}\left(\mathrm{r}_{i j}\right) \frac{\Theta_{i j}}{\sqrt{\Delta t}} \mathbf{e}_{i j},
$$




$$
\mathbf{F}_{i j}^{D}=-\gamma_{i j} w_{i j}^{D}\left(\mathrm{r}_{i j}\right)\left(\mathbf{v}_{i j} \cdot \mathbf{e}_{i j}\right) \mathbf{e}_{i j}
$$

where $\sigma_{i j}$ and $\gamma_{i j}$ are the controlling parameter for the strength of the random and dissipative force, respectively. $w_{i j}^{R}$ and $w_{i j}^{D}$ are the weight functions for the random and the dissipative force which go to zero after the cut-off distance. $\mathbf{v}_{i j}=\mathbf{v}_{i}-\mathbf{v}_{j}$ is the relative velocity and $\Theta_{i j}$ is a white noise with mean value of zero and variance of unity. By definition, the random and dissipative forces form the canonical ensemble in the calculation system and control the temperature. Espanol and Warren [3] showed that in order to satisfy the fluctuation-dissipation theorem, $\sigma_{i j}, \gamma_{i j}$ and the weight functions, $w_{i j}^{R}$ and $w_{i j}^{D}$, must be related and can be determined by

$$
\begin{aligned}
& \frac{\sigma_{i j}^{2}}{2 \gamma_{i j}}=k_{B} T \\
& w_{i j}^{C}\left(\mathrm{r}_{i j}\right)=w_{i j}^{R}\left(\mathrm{r}_{i j}\right)=\left[w_{i j}^{D}\left(\mathrm{r}_{i j}\right)\right]^{0.5}=w_{i j}\left(\mathrm{r}_{i j}\right)=\left\{\begin{array}{cc}
\left(1-\frac{r_{i j}}{r_{c}}\right) ; & r_{i j} \leq r_{c} \\
0 ; & r_{i j} \geq r_{c}
\end{array}\right.
\end{aligned}
$$

For simplicity, the conservative and random weight functions, $w_{i j}^{C}$ and $w_{i j}^{R}$ are considered as a linear function of the inter-distance between any pair of particles which vanishes at the cut-off distance $r_{c}$. Modified Velocity-Verlet (M-VV) algorithm proposed by Groot-Warren [2] and time steps of the size $0.01 \sqrt{\frac{m r_{c}^{2}}{k_{B} T}}$ was employed as the time integration method in all the simulations. The traditional value of 1.0 was used for the cut-off distance in all the calculations. Each condition was simulated using 3000 DPD particles with a number density of 3.0, (resulting in a fixed calculation box size of $10\left[\mathrm{r}_{\mathrm{c}}\right]$ in each direction) for $10^{7}-3 \times 10^{7}$ time steps, where the first $10^{6}$ steps were not included in time averaging calculations, as the system was assumed to reach its equilibrium state during that time. One can establish a dimensional analysis relating dimensionless parameters in DPD to real physical units which depends on the coarse-graining level. Table 1 deals with this conversion between DPD units used in this study and real physical units similar to one proposed and used by Ghoufi and Malfreyt [22]. 
Table 1: DPD and real units conversion for $r_{c}, m, \rho, \delta$, and $\eta$ which are cut-off distance, mass, density, time step, and viscosity respectively. $V$ is the volume of a water molecule (30 $\AA$ ), $M$ is the molar weight of water $\left(18 \mathrm{grmol}^{-1}\right), \mathrm{N}_{A}$ is the Avogardo's number, $k_{B}$ is Boltzmann constant, and T is temperature at 298 K.L,M,P, $\boldsymbol{\tau}, \boldsymbol{H}$ are length, Mass, density, time, and viscosity in the real units.

\begin{tabular}{cccc} 
DPD & \multicolumn{2}{c}{$\boldsymbol{D P D} \rightarrow$ real units } & Physical units \\
\hline bead & 1 & $\mathrm{Nm}$ & $3 \mathrm{H} 2 \mathrm{O}$ \\
$r_{c}$ & 1 & $\boldsymbol{L}:(\rho \mathrm{NmV})^{1 / 3}$ & $6.45[\AA]$ \\
$m$ & 1.00 & $\boldsymbol{M}: N_{m} M / N_{A}$ & $8.98 \times 10^{-23}[\mathrm{~g}]$ \\
$\rho$ & 3.00 & $\boldsymbol{P}: \rho N_{m} M / N_{A} r_{c}{ }^{3}$ & $\sim 996.3\left[\mathrm{~kg} \mathrm{~m}^{-3}\right]$ \\
$\delta t$ & 0.01 & $\boldsymbol{\tau}: \delta t m^{0.5} r_{d} /\left(k_{B} T\right)^{0.5}$ & $\sim 1[\mathrm{ps}]$ \\
$\eta\left(\gamma=4.5, k_{b} T=1.0\right)$ & $0.85 \pm 0.1$ & $\boldsymbol{H}: \eta \tau k_{B} T / r_{c}{ }^{3}$ & $0.001[\mathrm{~Pa} . \mathrm{s}]$
\end{tabular}

\section{Boundary conditions}

Perhaps one of the most widely-used boundary condition methods for simulation of fluids under non-equilibrium condition (mainly shear) is the well-known Lees-Edwards boundary condition [23]. The method was initially proposed (and always been successful) for molecular dynamics simulation of sheared fluids. The Lees-Edwards boundary conditions (L-E BC) introduces the desired velocity profile in any given calculation box by applying a constant velocity to the surrounding imaginary cells (with different signs for upper and lower neighboring cells). However, it has been shown that use of the original periodic L-E BC results in distorted velocity profiles which originates from the fact that the dissipative force in DPD uses relative velocity of a particle pair to calculate the force between them [7]. This is more pronounced when greater values of $\gamma$, the dissipative parameter, is used to reproduce higher Schmidt numbers. Different solutions have been proposed to address this issue for DPD simulations of sheared fluids. Chatterjee [7] proposed using so-called ghost particles in an additional layer at the borders of the central calculation cell. Some authors have been using a simple modification of the original scheme of LE $\mathrm{BC}$ that corrects the dissipative force by taking into account the velocity of the parent cells [24, 25]. This scheme is discussed in detail in a separate chapter and is called the Eulerian approach for the rest of this paper. This method will induce high deviations of the imposed shear rate from the measured value at low shear rates, which have been observed and reported in the literature as well [25-27]. Other methods that have been used resort to frozen particles and wall-bounded flows; e.g. Pivkin et al. [4] and Chen et al. [28]introduced the velocity profile by bounding the upper/lower cell borders with a moving wall in order to avoid direct interaction between two neighbor particles in opposite sides of the calculation box. However, using wall-bounded simulation cells results in additional effects, such as slip and density fluctuations near the wall. Although authors have been trying to address the density fluctuation issue [5, 
29] it should be considered that utilizing the approach of using frozen particles should be avoided unless the physics of confinement geometry needs to be addressed, in which case precaution is advised. In general there are three reasons for not including the frozen particles: i) Calculation times increase; ii) It violates the momentum conservation equation, since it does not hold for the interactions between solvent and wall particles; iii) Unlike MD, using frozen DPD particles as a coarse-grain level of MD does not provide any physical explanation unless meaningful coarse-graining procedures are followed, which is not the case for any of the proposed wall models. It should also be noted that using frozen particles has been the choice for most of steady shear studies via DPD. In the present work, we introduce a modification approach to the traditional L-E BC, and study the resulting velocity profiles, temperature control and pressure/stress measurements for each system.

\section{III.A Lagrangian approach}

One way to look at the DPD particles under flow is looking at them as individual momentum carriers that interact with each other in a reference coordinate moving with the flow field. To analyze the flow properties with DPD, one can look at the fluid parcels rather that a specific position in the flow field. In this paper, we propose both ways to look at the flow properties of the DPD fluid. In this section the Lagrangian approach, in which we analyze the flow in the reference coordinate attached to each fluid parcels, will be discussed. The Eulerian approach, in which the flow is analyzed in a reference coordinate system attached to the calculation cell, will be discussed in the next section.

DPD momentum carriers can start either from a stationary position or possessing an imposed initial velocity related to the set temperature (which reduces the time necessary to reach equilibrium state). After reaching the equilibrium state, each DPD carrier has a peculiar velocity and the ensemble has a velocity distribution, as shown in Figure 1. When any flow field is imposed on the calculation cell, e.g. shear, extension, or mixed state of shear and extension flow, DPD carriers will follow the field and it can be assumed that this velocity and the peculiar velocities of the DPD momentum carries can be superimposed.

Generally solving DPD equations involves using velocity-Verlet, VV, algorithm, which is described in detail in [2]. Due to the dependency of the dissipative force (DPD particle's acceleration) on its velocity and the dependency of the DPD particle's velocity on its acceleration, VV should be divided into two parts. In VV-Part-I particles' position and velocities are updated from the forces on the DPD particles and then forces on the DPD particles will be calculated based on updated velocities. In VV-Part-II the DPD velocities are corrected by the updated force values. In our proposed model, the flow field (shear) will be screened after VV-Part-I and the DPD particles will interact by their peculiar velocities; after updating the 
values for the peculiar velocities, the flow field will be superimposed as is depicted in the Figure 1. The detailed description of the method is discussed in Table 2.

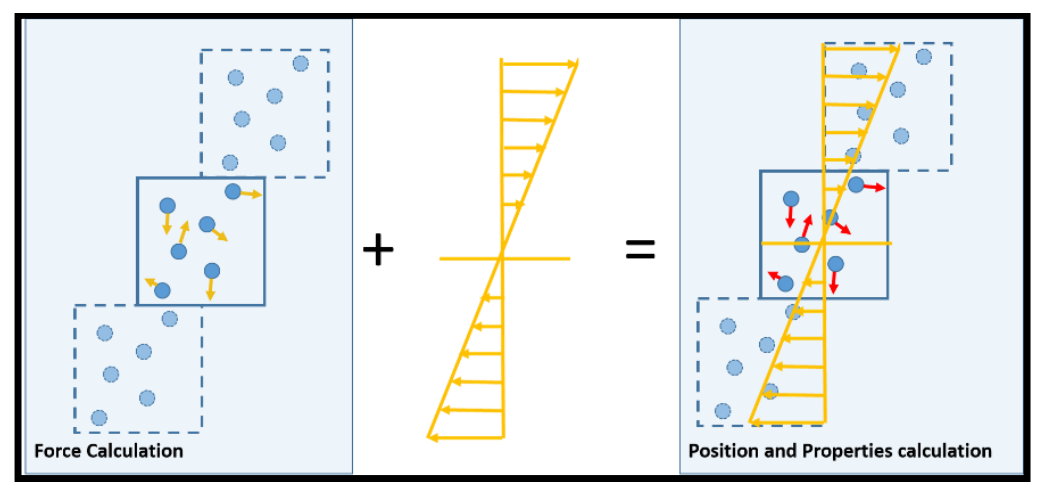

Figure 1: Schematic of the principals of Lagrangian approach

Table 2 Lagrangian approach incorporation in modified $V V$ algorithm to separate the flow from molecular motion

\begin{tabular}{|c|l|}
\hline VV_Part I & $\mathbf{r}_{\mathrm{i}}(\mathrm{t}+\Delta \mathrm{t})=\mathbf{r}_{\mathrm{i}}(\mathrm{t})+\Delta \mathbf{v}_{\mathrm{i}}(\mathrm{t})+\frac{1}{2}(\Delta \mathrm{t})^{2}\left(\frac{\mathbf{F}_{\mathrm{i}}}{\mathrm{m}_{\mathrm{i}}}\right)$ \\
\hline Force Calculation & $\mathbf{v}_{\mathrm{i}}(\mathrm{t})=\mathbf{v}_{\mathrm{i}}(\mathrm{t})-\mathbf{r}_{\mathrm{i}}^{y}(\mathrm{t}) \cdot \dot{\gamma} \quad \begin{array}{l}\text { Screening Flow field } \\
\text { VV_Part II }\end{array}$ \\
\hline$\tilde{\mathbf{v}}_{\mathrm{i}}(\mathrm{t}+\Delta \mathrm{t})=\mathbf{v}_{\mathrm{i}}(\mathrm{t})+\lambda(\Delta \mathrm{t}) \frac{\mathbf{F}_{\mathrm{i}}}{\mathrm{m}_{\mathrm{i}}}$ \\
\hline Flow Superposition & $\mathbf{F}_{\mathrm{i}}(\mathrm{t}+\Delta \mathrm{t})=\mathbf{F}_{\mathrm{i}}\left(\mathbf{r}_{\mathrm{i}}(\mathrm{t}+\Delta \mathrm{t}), \tilde{\mathbf{v}}_{\mathrm{i}}(\mathrm{t}+\Delta \mathrm{t})\right)$ \\
\hline
\end{tabular}

\section{III.b Eulerian approach}

The simplest modification to the traditional L-E BC can be applied by modifying the dissipative force between two neighboring particles located on opposite sides of the calculation box. One would need to move the upper and lower neighboring cells with the velocity of $V_{\text {Cell }}^{\text {Upper }}=-V_{\text {Cell }}^{\text {Lower }}=\frac{\dot{\gamma} L_{y}}{2}$, to obtain the shear rate of $\dot{\gamma}$ for a calculation box of $\left(L_{x}, L_{y}, L_{z}\right)$ size. A schematic view of the imposed velocity is shown in figure 2. 


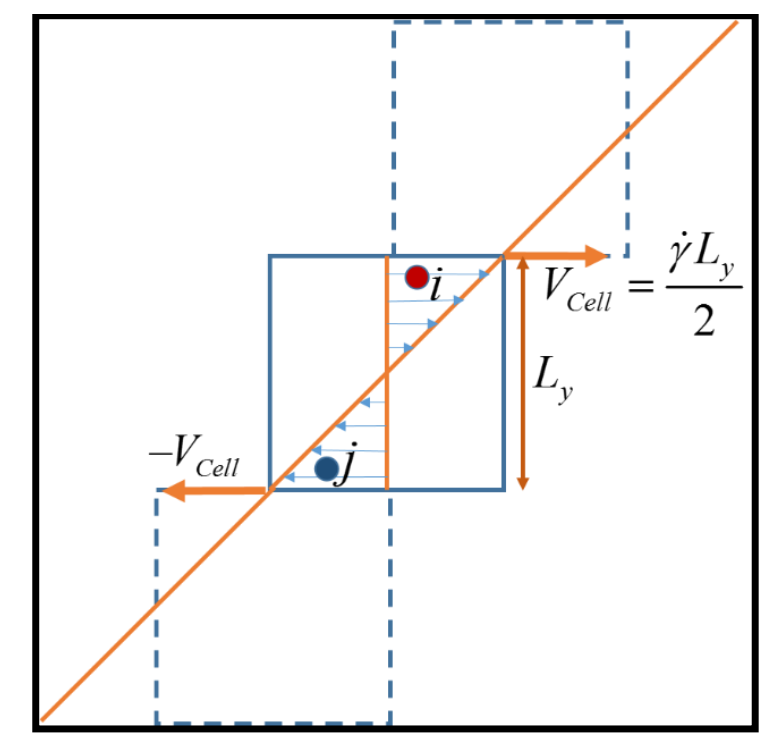

Figure 2. Schematic view of Lees-Edwards boundary conditions

While it should be noted that the calculation cell is fixed in this approach, we focus on the calculation of dissipative force between particles $i$ and $j, F_{i j}{ }^{D}$, and more specifically the relative velocity of these particles, $v_{i j}$, interacting from opposite sides of the calculation box. In order to take into account the effect of continuous velocity profile, in addition to the periodicity of the system, we now change the dissipative force to the following form:

$$
\mathbf{F}_{i j}^{D}=-\gamma_{i j} w_{i j}^{D}\left(\mathrm{r}_{i j}\right)\left[\mathbf{e}_{i j} \cdot\left(\mathbf{v}_{i}-\mathbf{v}_{j}-2 \mathbf{V}_{C e l l}\right)\right] \mathbf{e}_{i j}
$$

Figure 3 shows the steady velocity profiles at different shear rates developed using abovementioned procedure for Lagrangian method. The velocity profiles reveal that a wide range of shear rates can be obtained using this approach. It should be noted that results in Figure 3 are measured for a dissipative force parameter of $\gamma=4.5,50$ averaged over 500 steps. Same results are obtained for Eulerian approach that are not shown here. 

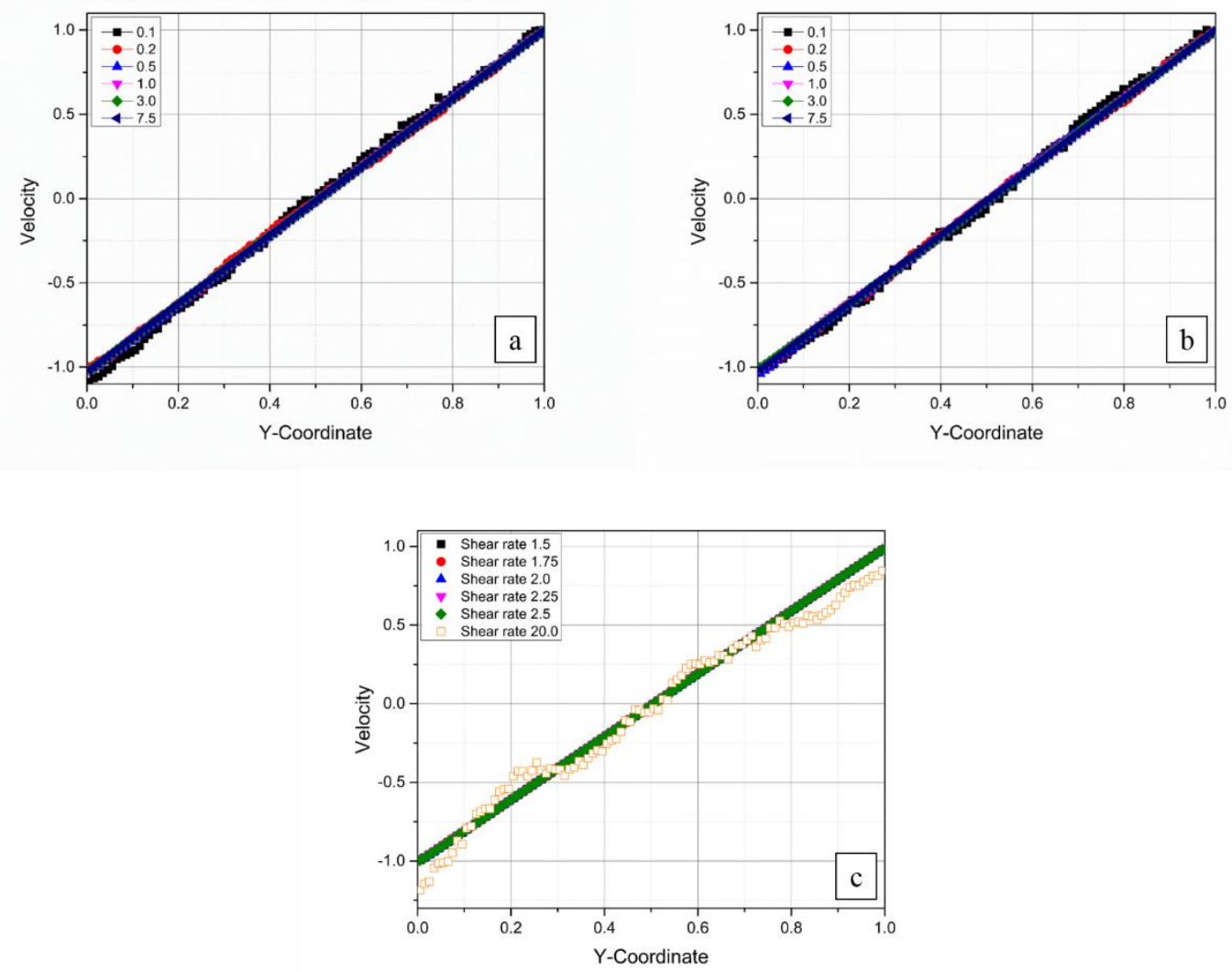

Figure 3. Normalized velocity profiles developed using Lagrangian approach $(a) \gamma=4.5$ and $(b) \gamma=50$ for a wide range of shear rates. (c) Break down of the velocity profile at large shear rate (20.0) for $\gamma=4.5$.

One can calculate temperature of a system by measuring ensemble average kinetic energy of the particles, which can be determined by

$T=\frac{1}{3 N} \sum_{i=1}^{N} m_{i}\left(\mathbf{v}_{i}-\mathbf{u}\left(r_{i}\right)\right) \cdot\left(\mathbf{v}_{i}-\mathbf{u}\left(r_{i}\right)\right)$

The term $u\left(r_{i}\right)$ corresponds to the value of the velocity (from the velocity profile) at the position of $i$-th particle in the velocity gradient direction, where $v_{i}-u\left(r_{i}\right)$ is the peculiar velocity of the particle $i$-th, $m_{i}$ is the mass of a DPD particle and $N$ is the total number of particles. Figure 4 shows the temperature controlling effect of the modified dissipative force for the Eulerian and Lagrangian approaches, using dissipative parameters $(\gamma=4.5,50.0)$.

An increase of the temperature under strong non-equilibrium conditions is a well-known phenomenon that has been studied extensively and different thermostats have been proposed to address the issue $[8,9]$. As one can clearly conclude from the Figure 4, the dissipative force with higher strengths (higher 
dissipative parameter) can control the temperature up to higher shear rates. Although it is generally accepted that the use of large friction parameters, requires smaller time steps for better temperature stability, we have run simulations with a wide range of $\Delta t(0.0001-0.1)$ and found that the time step does not affect the temperature stability while the noise to signal ratio increases by decreasing the time step. In contrast to a better temperature controlling effect, it was reported [7] that using higher dissipative parameters will result in a distorted velocity profile due to limitations of the L-E boundary conditions. However, the velocity profiles for the dissipative parameter of $\gamma=50.0$ plotted in Figure $3 \mathrm{~b}$ shows that the Lagrangian approach constructs the correct velocity profile and consequently correct shear rate.
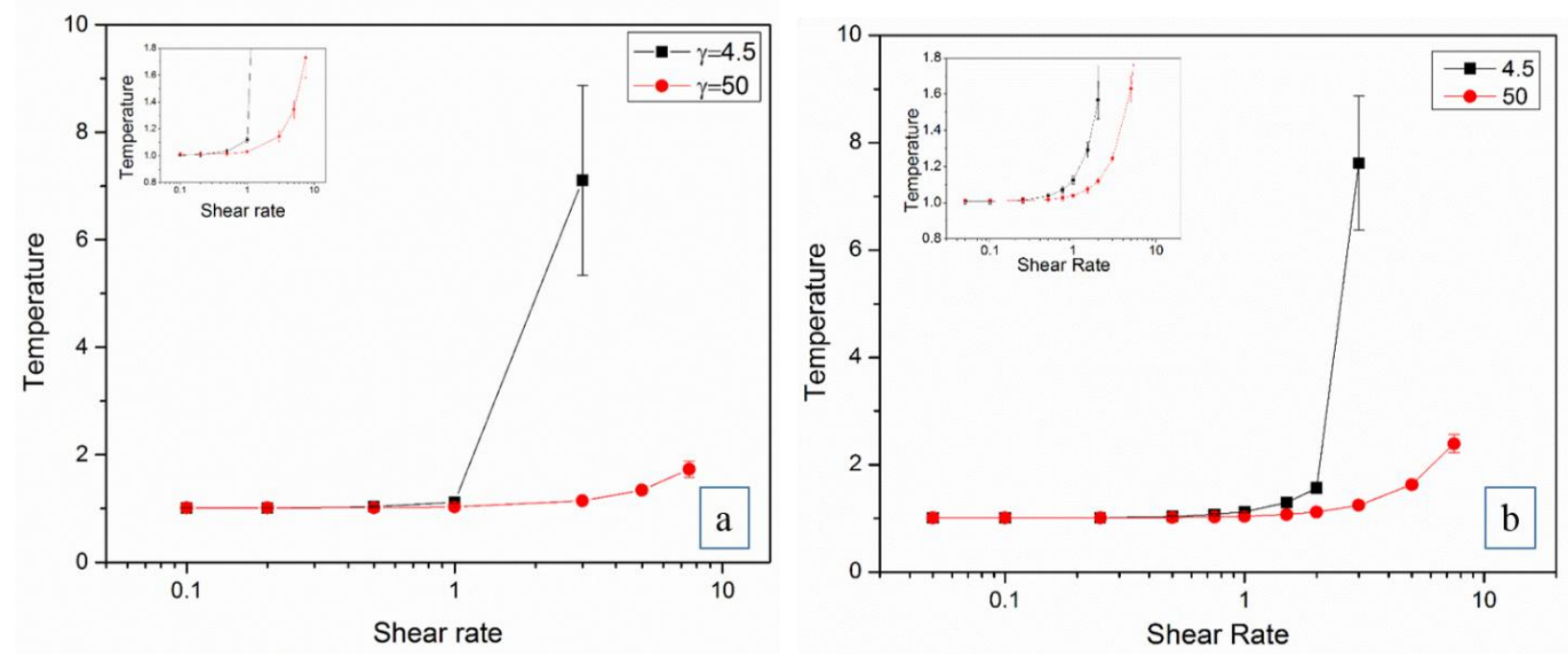

Figure 4.Temperature controlling effect of the proposed dissipative force as a function of shear rate. (a) Lagrangian, (b) Eulerian.

To explain the better performance of the higher values of dissipative parameter in controlling the temperature increase, we monitored the velocity distribution of particles at each shear rate and gamma. Theoretically, the velocity distribution of a particulate system at any time step has to follow MaxwellBoltzmann distribution, where parameters of this function will directly relate to the temperature. Figure 5 shows the velocity distribution of DPD particles, for three different shear rates using a range of dissipative parameters for both Eulerian (a-c) and Lagrangian (e-g) approaches. Three components of the velocity vector will be affected by presence of the shear field. However, the components in the velocity gradient and vorticity directions show $5 \%$ deviation when compared to equilibrium. For this reason only the component in the shear direction is depicted in the figure 5.

It reveals two important behaviors of DPD particles under shear: firstly, regardless of the dissipative parameter value, as the particles undergo stronger shear rates, the velocity distribution curve is broadened 
and thus the temperature increases, and secondly, higher the dissipative parameter will results in a more stable velocity distribution at higher shear rates.
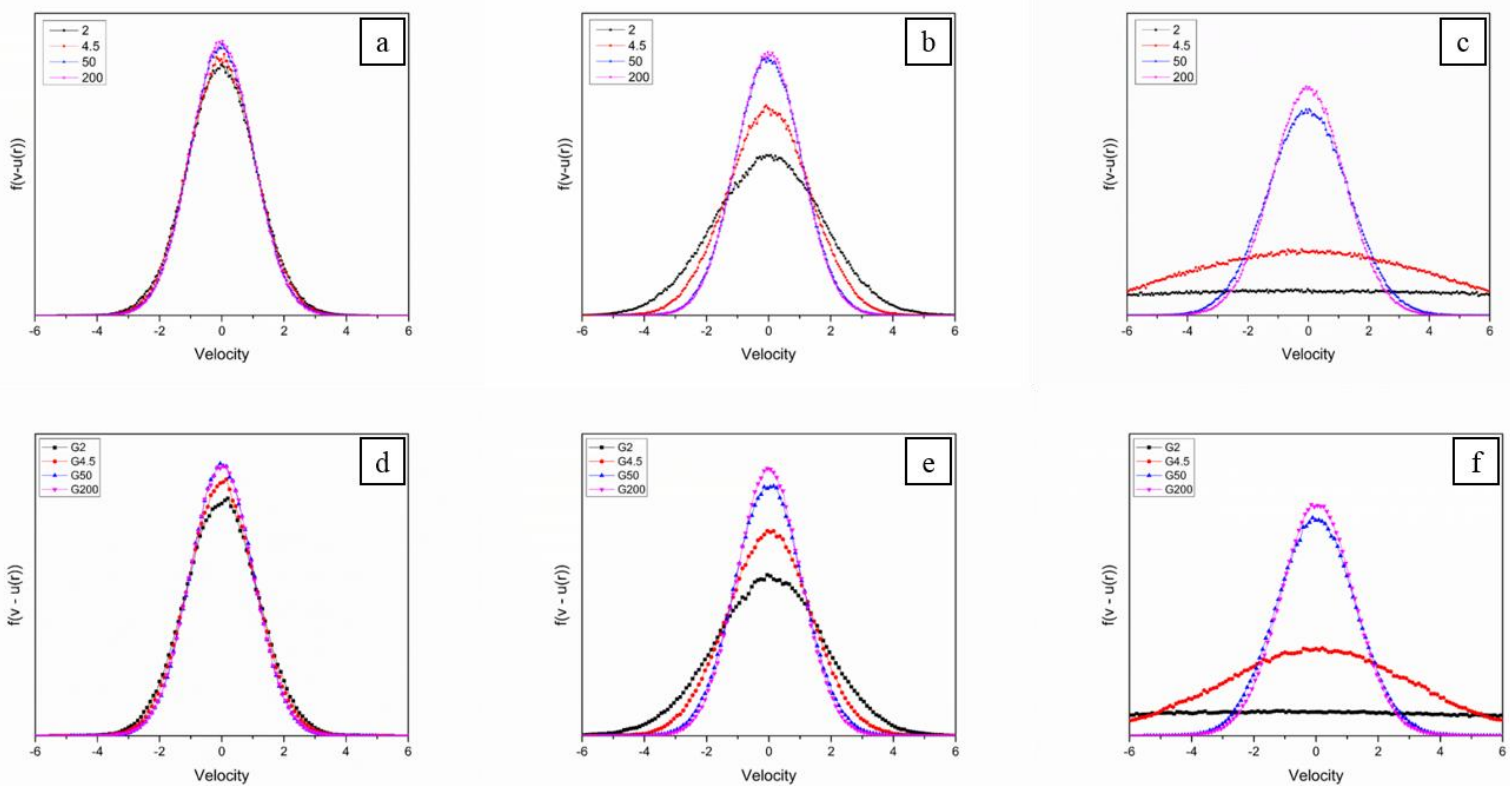

Figure 5. Velocity distribution for different values of dissipative parameter (the legend), for shear rates of: Eulerian approach a)1.0, b)2.0 and, c)5.0 and Lagrangian approach e) 1.0, f)2.0, g)5.0.

Since different values of dissipative parameter result in different temperature and velocity distribution curves at various shear rates, in the next section a complete study of shear viscosity as a function of shear rate, $\dot{\gamma}$, and dissipative parameter, $\gamma$, is presented. This study is divided into two groups: First, we measure the shear viscosity from the microscopic (molecular) definition of stress; this will be covered in section IV. Secondly, it will be determined from fitting procedures e.g. Poiseuille flow (PF) and transient shear flow; this will be covered in section V. In this paper, we introduce the Lagrangian approach and explore its advantages and disadvantages, and, for the first time, we systematically use all possible procedures for measuring viscosity in DPD to give a better insight in capability of DPD in dealing with non-equilibrium flows and rheological studies. We hope the results presented in this paper can be a guide to authors willing to use particulate simulation systems especially DPD for rheological studies that suit their system. 


\section{Microscopic definition of Stress}

\section{IV.a Steady Shear Viscosity}

Given the forces between each pair of particles, and the velocity of individual particles, one can calculate the pressure tensor for a DPD system by

$$
\mathbf{P}=\frac{1}{V}\left\{\sum_{i=1}^{N} m_{i}\left(\mathbf{v}_{i}-\mathbf{u}\left(r_{i}\right)\right) \otimes\left(\mathbf{v}_{i}-\mathbf{u}\left(r_{i}\right)\right)+\sum_{j>i}^{N} \sum_{i=1}^{N-1} \mathbf{r}_{i j} \otimes \mathbf{F}_{i j}^{T}\right\},
$$

where $\otimes$ is the dyadic product of the two vectors. The stress tensor would be the negative of the pressure tensor $(\mathbf{S}=\mathbf{- P})$, and thus the shear viscosity of a DPD fluid can be calculated from the $x y$ component of the stress tensor:

$$
\eta=\frac{\left\langle S_{x v}\right\rangle}{\dot{\gamma}}
$$

The bracket in (eq.11) represents the time average value of the shear stress.

Although in calculation of the stress (eq.10) all the components of the forces should be considered, it should be noted that for stress calculation in the Lagrangian approach, stresses arising due to the flow field are neglected.

By careful review of the approach in Table 2, one can see that the force calculation is done in the stage that there is no flow present and stress of the flow field should be calculated separately and be added to the stress calculated form particles' interactions. For this reason we calculated the flow stress though the dissipative tensor proposed by Marsh et al.[30]:

$$
\Pi_{D}=-0.5 m \rho^{2} \gamma \int d \mathbf{R} w(\mathbf{R})(\mathbf{e} \otimes \mathbf{e})(\mathbf{R} \cdot(\mathbf{u}(\mathbf{r})-\mathbf{u}(\mathbf{r}-\mathbf{R})))
$$

where $u(R)$ is the velocity of the flow at the position of $R$. Viscosity measurement data for Eulerian (eq.10) and Lagrangian approach (eq.10,12) are depicted in fig.6. As it can be seen from the graphs both method predict the same values and dependency of the viscosity to the dissipative parameter and shear rate. It should be noted that the minimum accessible shear rate for Lagrangian is lower and signal to noise ratios are higher compare to Eulerian approach.

The limitation of Eulerian approach can be explained through the physical concept behind the LE BC. Owing to the fact that velocity gradient in Eulerian method requires diffusion throughout the calculation cell through imaginary cells at the top and bottom of the calculation cell, this method is always limited by the lower values of strain rates. This phenomena can be illustrated in a numerical example: Consider a 
calculation cell of a size used in all simulations of this study; $10\left[\mathrm{r}_{\mathrm{c}}\right]$ in each direction. We want to study the viscosity of the system by applying a shear rate of 0.01 . Knowing that maximum velocities are occurring at the top and bottom of the calculation cell ( $5 \times 0.01=0.05)$ and the fact that all the simulations are running at the temperature $(\mathrm{KbT}=1.0)$ we can calculate the share of the shear field compare to the mean velocity of the particle due to its temperature; which is $5 \%$. This small share will hinder the diffusion of momentum into the calculation cell. In fact our data showed that while Eulerian approach is limited to shear rate 0.05 velocity profile can be reproduced for shear rate of 0.005 for Lagrangian approach. This discrepancy between the applied shear rate and the measured shear rate is the reason for the increase in the viscosity at the low shear rates (result are not shown here). This is a very important shortcoming of Eulerian approach that is neglected or has not been studied in detailed in some literature using DPD at low shear rate studies [17, 25].

It should be noted that an unphysical shear-thickening behavior is observed at high shear rates for both Eulerian and Lagrangian approaches when a small value of dissipative parameter is employed. This can be explained through equation 9. The first term on the right hand side of the pressure tensor, i.e., the kinetic part, is a function of particle velocity. As explained before, the deviation of particle velocities at high shear rates (which gives rise to temperature deviation) is reflected in this part of the equation.
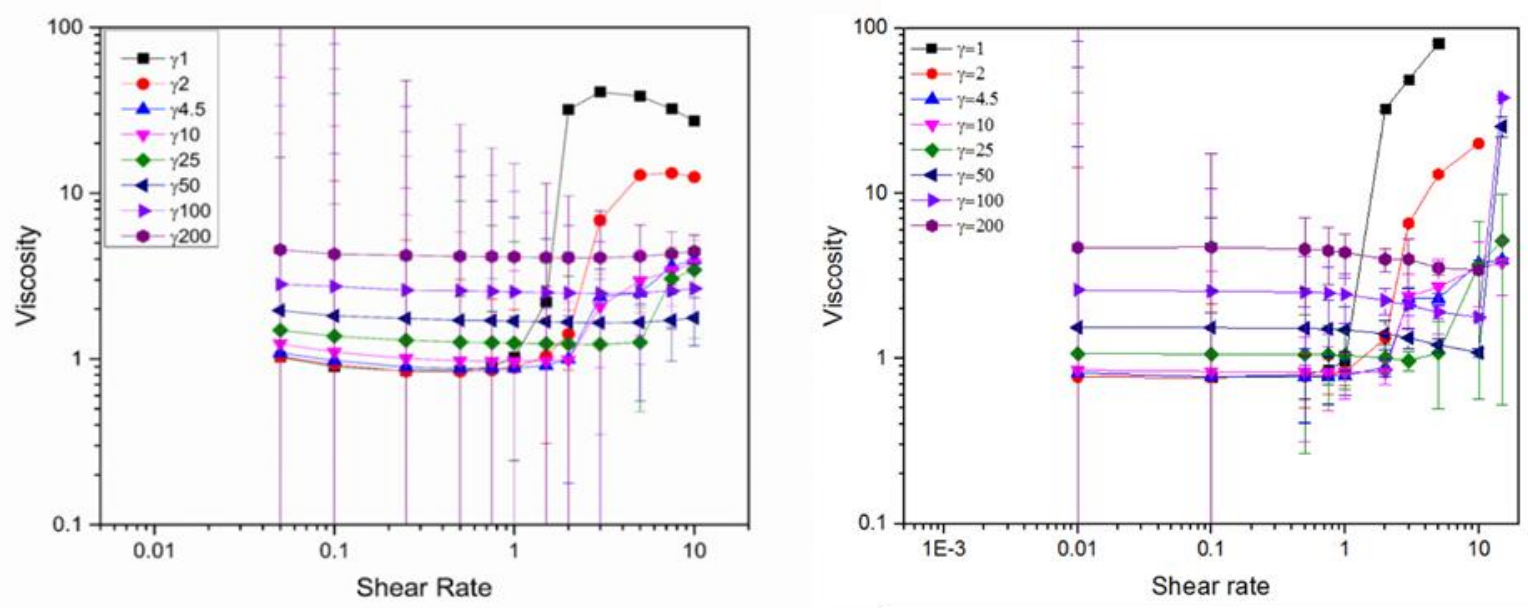

Figure 6: Shear viscosity of a DPD fluid as a function of shear rate for a range of dissipative parameters for (left) Eulerian and (right) Lagrangian

Thus, an increased velocity (meaning increased temperature) results in increased viscosity. It should be also noted that if one tends to measure viscosity or other material functions e.g. first normalstress coefficients, with the LE- BC (Eulerian approach) at low shear rates (less than 0.05), the shear flow field is not stably developed even after $10^{6}$ steps (results are not shown here) and thus, calculating the viscosity based on the applied shear rates (not the measured one) will lead to erroneous high values. We 
would like to reiterate the fact that by using Lagrangian approach and separating the material stress and flow stress, viscosities can be measured at lower shear rates with a higher signal to noise ratios.

Figure 7 shows the temperature calculated for a wide range of shear rates and dissipative parameters at which the viscosity was calculated. As explained in the previous section, this temperature rise is correlated with the ability of the dissipative force to maintain the velocity distributions. From the temperature deviation and viscosity measurements, we proposed a simple guideline that should be followed in order to bypass any effect of temperature raise on the viscosity measurement. At any given dissipative parameters, the maximum strain rate should be limited to the critical shear rate by:

$$
\dot{\gamma}<\dot{\gamma}_{\text {critical }} \sim \gamma^{0.65}
$$

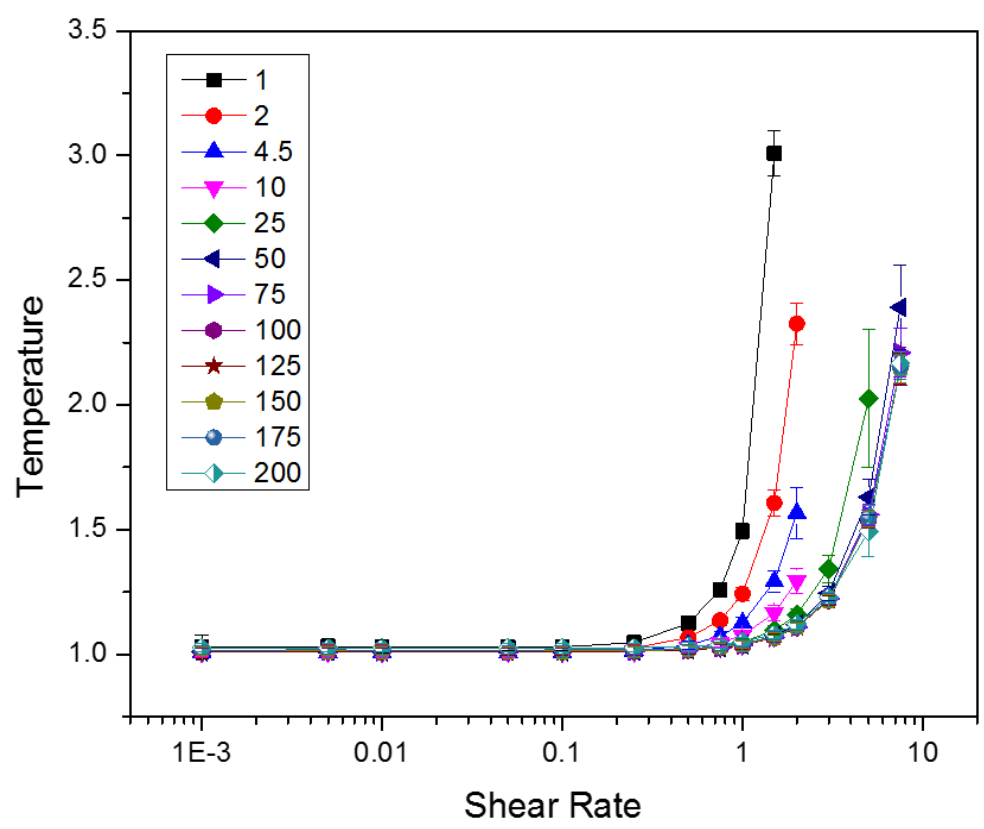

Figure 7. Temperature as a function of shear rate for a range of dissipative parameters.

Different shear viscosity values at the same shear rate for different dissipative parameters could originate in the fact that the velocity distribution changes from one $\gamma$ value to another. We have also used the Lowe-Anderson (LA) thermostat coupled with DPD thermostat, although the results are not shown here. We concluded that coupling the DPD thermostat with the LA method significantly changes the dynamics of the system. Despite the fact that the LA scheme can effectively control the temperature, at the same time it makes the results incomparable to the ones obtain by regular DPD. Obviously, in the case of the stand-alone LA time integration scheme, which does not include the dissipative and random forces, there is no friction parameter dependence to be studied and the fluid dynamics is solely controlled by the bath probability function introduced in the LA. 
Considering the distribution curves in Figure 6, one would expect the velocity distributions at equilibrium conditions to be the same for any given dissipative parameter. Figure 8 shows the velocity distributions for a range of dissipative parameters. Since figure 9 confirms that all different values of dissipative parameter yield equal velocity distributions at zero-shear limit, in the next section we present zero-shear viscosity measurements of the same system for a range of dissipative parameters and temperatures.

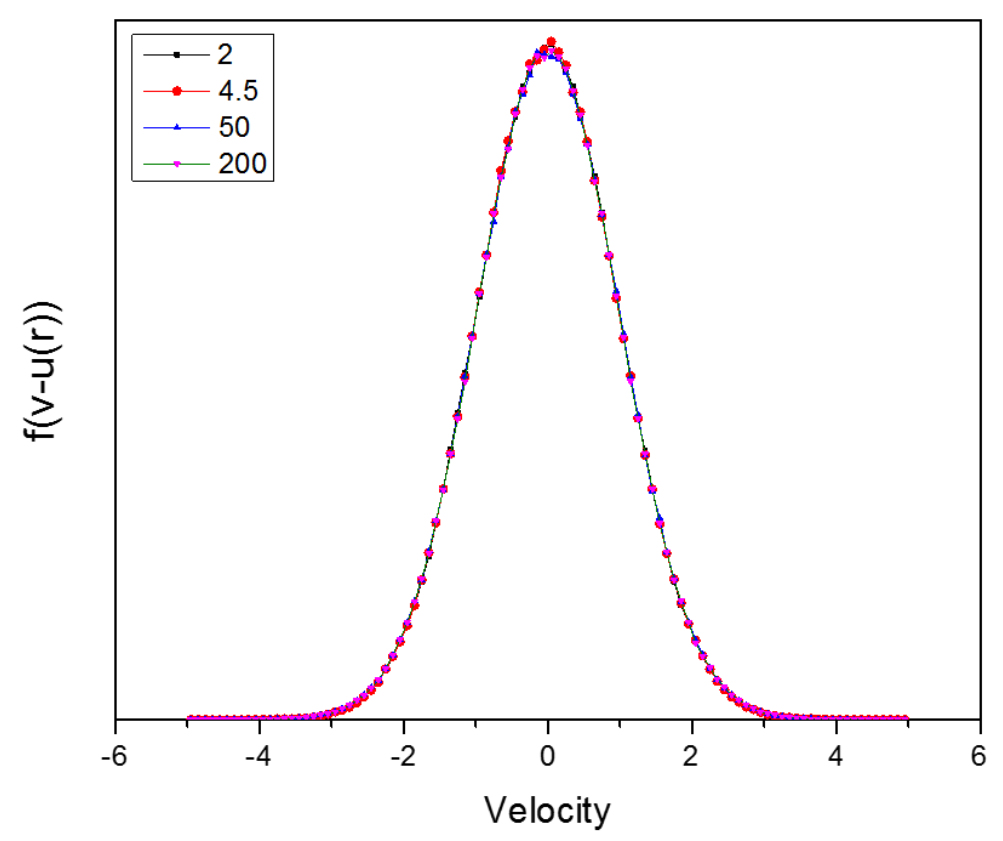

Figure 8. Velocity distribution for different dissipative parameters at equilibrium condition.

\section{IV.b Zero shear viscosity}

With the pressure tensor at hand, one can calculate the zero-shear viscosity of a given system, using Green-Kubo expression of pressure auto-correlation function:

$$
\eta=\frac{V}{3 k_{B} T} \int_{0}^{\infty} d t\left\langle P_{\alpha \beta}(t): P_{\alpha \beta}(0)\right\rangle ;(\alpha \neq \beta),
$$

In practice the simulations were ran for $3.1 \times 10^{7}$ time steps were the first $10^{6}$ steps were not included in the results, assuming that the system reaches its equilibrium state during this time. Pressure autocorrelation function was calculated over 40,000 time origins, and the result were integrated out for 2000 time steps, $t^{*}=20$. According to equation 3, there is a direct relationship between the conservative force parameter and temperature of a DPD system $\left(a_{i j}=25 k_{B} T\right.$, considering a constant compressibility 
and number density of 3.0). However, based on the expression for the calculation of the pressure tensor (equation 9), increasing the temperature will increase the first term on the right hand side of the equation (kinetic part), and adapting equation 2 for conservative force will increase the second term as well (virial part). Increase in shear viscosity by increasing the maximum repulsion parameter has been also observed in the literature [19].These two effects together will increase the viscosity of a DPD system as a function of temperature, which is in contradiction with the standard temperature dependence of the shear viscosity. Figure 9 shows this behavior for a range of dissipative parameters and two different conservative forces: one calculated by an adaptive approach, where the conservative parameter has been adapted following the equation 3, and one by a non-adaptive approach, where the conservative force has been kept constant, for different temperatures. The results in figure 9 proves that when an adaptive scheme is followed for the conservative force parameter, the zero shear viscosity increases by the kinetic temperature. On the other hand, the non-adaptive approach reproduces physically meaningful results in regards to temperature dependence of the viscosity. It is important to note that the using non-adaptive approach does not violate any physical laws. Although one can argue that the systems with different temperatures and the same conservative force differ in their chemical identity, presence of a minimum required conservative force prevents the system to become compressible. As it is stated in the recent publication by Pan et al. [18] DPD fluids are susceptible to compressibility issues. They have shown that DPD fluids can be considered (nearly) incompressible under 3 conditions: 1- small mass of DPD particles, 2- high temperature in systems with no conservative interactions, and finally increasing the repulsive force higher than the value predicted by GW scaling relation. Backer et al. [6] performed similar study to compare different methods for viscosity measurements, however they did not use the conservative force at the temperature of 0.5 which results in a compressible fluid. Marsh et.al. [30] suggested different dissipative parameter dependence for the viscosity of a DPD fluid

$$
\eta=\frac{45 k_{B} T}{4 \pi \gamma}+\frac{2 \pi \rho^{2} \gamma}{1575}
$$

Equation 13 predicts a rather steep decrease in the viscosity as the dissipative parameter increases, followed by an increase; ultimately, the viscosity becomes independent of temperature at high dissipative parameters. Considering the fact that equation 13 is written for a DPD fluid without conservative force, this behavior is a direct consequence of having a compressible fluid. Although the viscosity increases by increasing the dissipative parameter at all temperatures for the adaptive method as expected by the abovementioned expression, our simulations show different trends at different temperature regimes when the non-adaptive approach is used. At low temperatures $(<1.0)$, the viscosity increases almost linearly with the dissipative parameter. At unity $\left(k_{B} T=1.0\right)$, which is the temperature of the choice for majority 
of DPD simulations, the viscosity is in agreement with the analytical predictions. At high temperatures $(>1.0)$, and where a low dissipative parameter is used, the viscosity starts to rise and eventually at very high temperatures, the viscosity of a DPD fluid with dissipative parameter of 1 becomes larger than the one with $\gamma=100$. This can also be explained by equation 13 , as at the low dissipative parameter values, the first term on the right hand side of the equation is the dominant factor (diffusive), which predicts a linear increase of the viscosity by the temperature. However, when the friction term is effectively increased, the second term on the right hand side dictates the viscosity of the fluid (dissipative term). Needless to mention that introduction of the conservative force based on the expression given by GrootWarren changes this scheme. Although in our case there is a conservative force the effect of conservative force can be interpreted through diffusion term. At low dissipative parameter increasing the maximum repulsion parameter will significantly decrease the diffusion term and consequently the compressibility of the liquid (non-adaptive approach) that is why the trend at low viscosity and low temperature is totally opposite. Also at high temperature and low dissipative parameter non-adaptive approach will lead to more diffusion dominated state and it can be seen through a sharp erroneous increase in the viscosity. On the other hand at low temperature and low dissipative parameter increase in the repulsive force will lead to the correct behavior in the case of non-adaptive and erroneous results from adaptive state that is due to the compressibility or high diffusion in the adaptive case.
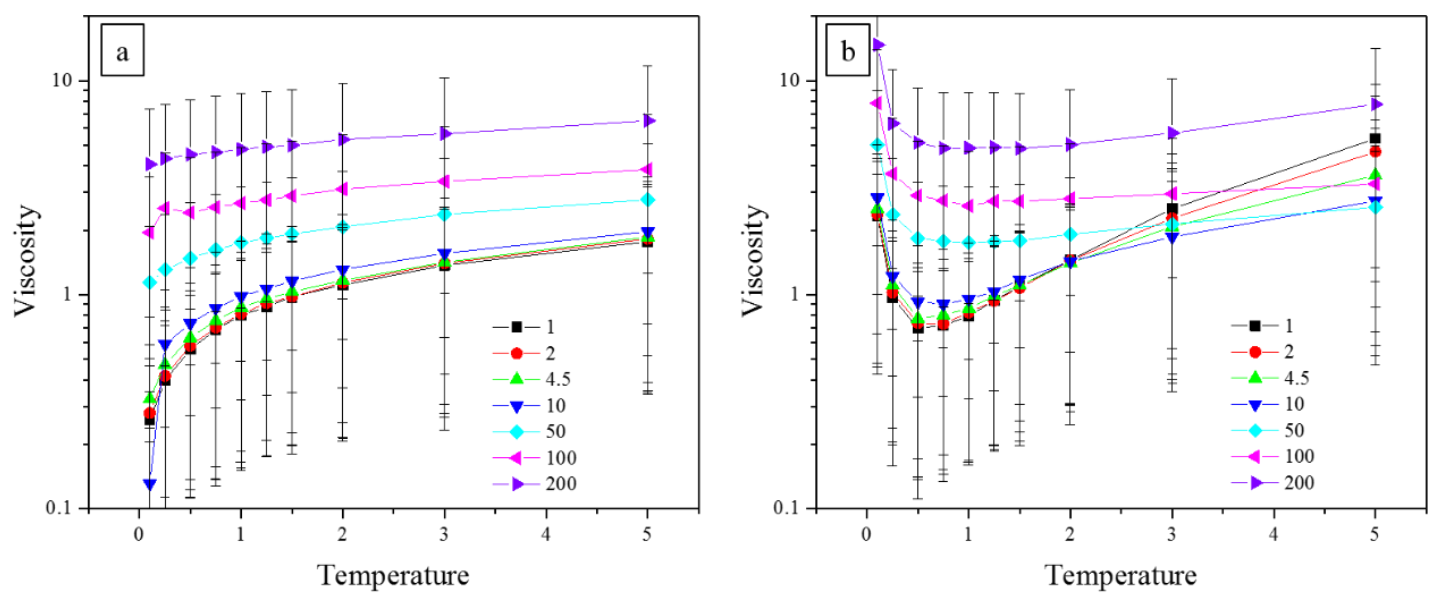

Figure 9 Zero shear viscosity as a function of temperature for different dissipative parameters and using: a) adaptive and, b) non-adaptive approach for the conservative force. 


\section{Numerical fitting procedures}

\section{V.a Poiseuille flow (PF)}

While measuring microscopic stresses is common in the literature as a way to measure viscosity in MD simulations and generally in particle-based simulation methods, numerical fitting is a powerful method to obtain viscosity of a medium both experimentally e.g. particle image velocimetry or Doppler optical coherence tomography, and by simulation methods. Two types of well-understood and characterized flow types for this purpose are Poiseuille flow, PF, and start-up shear transient flow. In this study, we perform a systematic analysis of the ability of both methods to measure viscosity in DPD fluids and compare to the first method presented in section IV. In this section PF will be discussed and in the next section start-up transient flow will be described as the methods to calculate viscosity.

There are two well-established methods to generate PF in DPD. The first method is the so-called periodic Poiseuille flow method (PPFM) [6, 31], in which the calculation cell is divided into two symmetric regions and a specific body force is applied to each DPD particle located in the top half of the calculation cell, while an equal force in the opposite direction is applied to the DPD particles in the other half of the calculation cell. The second method is wall-bound PF in which the fluid domain is confined by presence of the wall particles located at its boundaries, and a body force is applied to all solvent particles bound between the two walls. Different boundary conditions can be imposed to obtain no-slip at solidliquid boundaries, e.g. bounce-back, specular reflection, and Maxwellian [4, 29, 32-34]. Despite all the efforts, wall-bounded flows remains as one of the most controversial and unsettled issues in DPD and we will address this issue separately in another work. In this work, we only focus in PPFM to avoid any additional complexity arising from the solid-liquid interactions in DPD.

We use PPFM by applying a body force, $f_{b}=0.02$, to 24,000 DPD particles with a density of $\rho$ $=3 r_{c}^{-3}$, same parameter set used in Ref. [4], and velocity profile calculated through the calculation cell by discretizing the calculation cell into 100 bins and performing averaging over each bin in 100 time steps. The results are shown in Figure 10 for different values of dissipative parameters. 


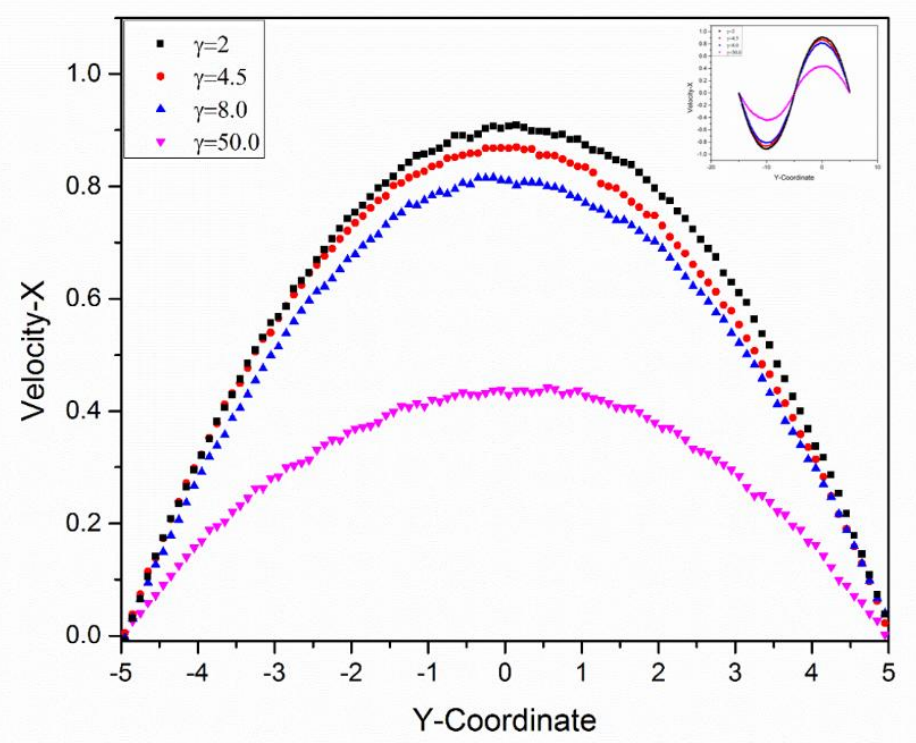

Figure 10: Velocity profile for Poiseuille flow in PPFM, inset shows the complete periodic velocity profile across the channel

Solving the Navier-Stokes equation for a rectangular channel under pressure gradient equal to $-\frac{\partial P}{\partial x}=\rho f_{b}$ yields [6]:

$$
V_{x}(\mathrm{y})=V_{\text {center }}\left(1-\left(\frac{y}{d}\right)^{2}\right)
$$

$\mathrm{V}_{\text {center }}$ is proportional to channel depth and inversly proportional to the viscosity of the fluid.

$$
V_{\text {center }}=\frac{L_{y}}{2 \mu}\left(-\frac{\partial P}{\partial x}\right),
$$

The values of viscosity and channel depth can be calculated from velocity fitting. The channel depth value is obtained to verify the fitting procedures. The values for density, dissipative parameter viscosity, and channel depth are shown in Table 3. It should be noted that the maximum deviation of all the measurements was less than $1 \%$. 
Table 3: Fit values for measuting viscosity from velocity profile for Poiseuille flow (PF)

\begin{tabular}{c|ccccc}
$\begin{array}{c}\text { Gamma } \\
{[\boldsymbol{\gamma}]}\end{array}$ & $\begin{array}{c}\text { Density } \\
{[\boldsymbol{\rho}]}\end{array}$ & R-squared & $\begin{array}{c}\text { Cell-Size } \\
{[\mathbf{L x}]}\end{array}$ & $\begin{array}{c}\text { Viscosity Marsh } \\
{[\boldsymbol{\mu} \text { Marsh }]}\end{array}$ & $\begin{array}{c}\text { Viscosity PF } \\
{[\boldsymbol{\mu} \text { PF }]}\end{array}$ \\
\hline $\mathbf{2}$ & 3.00 & 0.999 & 5.02 & 1.862 & 0.831 \\
$\mathbf{4 . 5}$ & 3.00 & 1.000 & 5.00 & 0.957 & 0.866 \\
$\mathbf{4 . 5}$ & 6.00 & 1.000 & 4.98 & 1.442 & 1.641 \\
$\mathbf{8}$ & 3.00 & 1.000 & 5.00 & 0.735 & 0.925 \\
$\mathbf{5 0}$ & 3.00 & 0.998 & 5.00 & 1.867 & 1.712 \\
$\mathbf{5 0}$ & 6.00 & 0.998 & 5.00 & 7.252 & 6.059 \\
\end{tabular}

Based on equation 13, which is known as the Marsh equation, the microscopic viscosity is composed of two parts. Although this equation has been derived for a system without conservative force, one can consider it as a guide line to expand it to the case with conservative force. The first part is the kinetic contribution, which is proportional to temperature and inversely proportional to $\mathrm{t}$ dissipative parameter that is related to the particle diffusion. This part of the viscosity resembles the viscosity of gases. The second part, which is a dissipative contribution, is related to the friction between particles and is dominant at higher density and/or higher values of dissipative parameter. This part resembles more the viscosity for liquids. For this reason, we performed the viscosity measurement in a range of density and dissipative parameter to assess how accurate the Marsh equation is in predicting viscosity values, and how reliable is to measure the viscosity with fitting methods such as PPFM.

As can be seen through Table 3, the results from PPFM qualitatively follow the Marsh values of viscosity except at low values of the dissipative parameter, which can be related to domination of the diffusive contribution over the dissipative contribution. Over-prediction of the Marsh equation has been reported in other works as well [35-37]. However, by looking at the shear rate (stress) level across the channel in Figure 11 for two dissipative parameters and densities, it can be seen that at low dissipative parameter the stress/strain-rate level does not change with density, while at higher levels of the dissipative parameter it will. This can be explained by the Marsh equation, in which second term, frictional term, is dominant at higher values of dissipative parameter for higher densities ( $\rho^{2}$ pre-factor). 

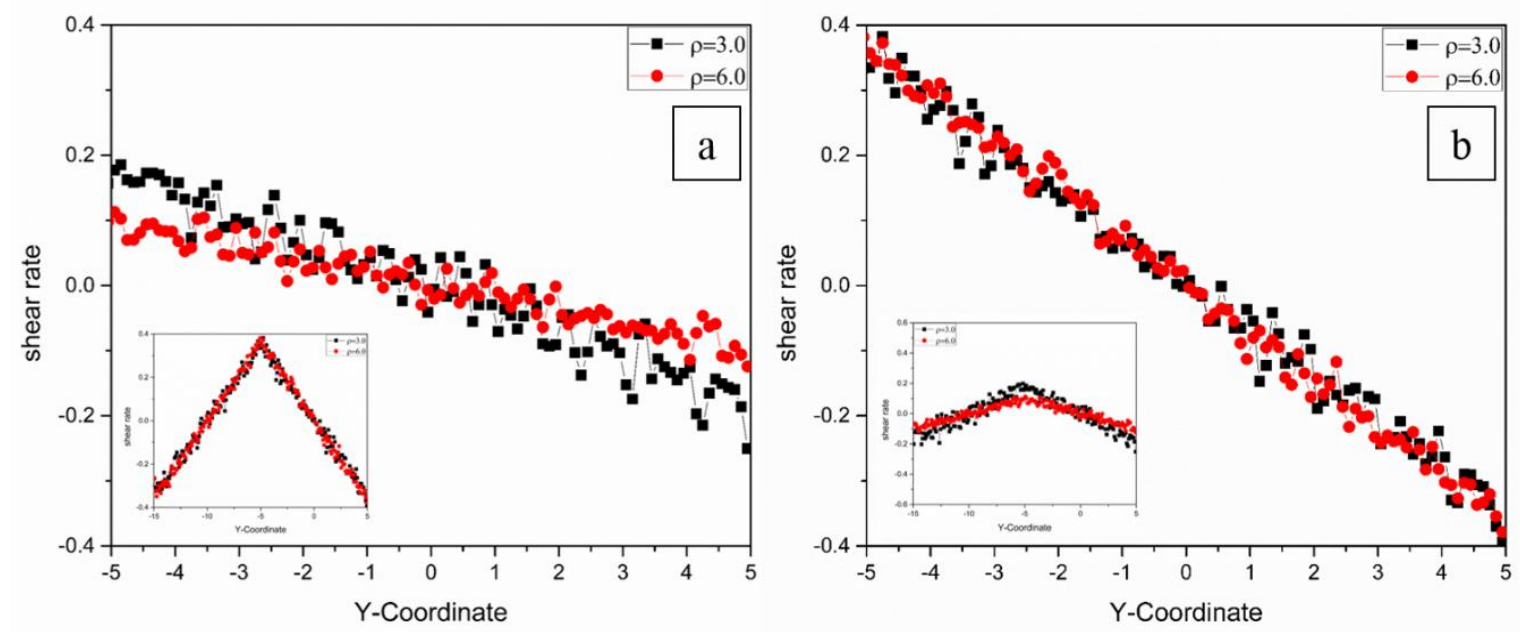

Figure 11: Shear-rate profile through the flow channel for different densities and dissipative parameter $(\gamma)($ a) $50,(b) 4.5$

\section{V.b Transient shear viscosity}

In this section, the viscosity measurement is performed by another fitting technique based on start-up transient shear flow. Solving the momentum diffusion equation in $1 \mathrm{D}\left(\partial_{t} V_{x}=v \partial^{2}{ }_{y} V_{x}\right)$, can provide a methodology to obtain the kinetic viscosity and respectively dynamic viscosity of the DPD fluid. Start-up transient shear flow has been employed by other authors to measure viscosity of particlebased simulation techniques and the procedures used to obtain the viscosity is discussed elsewhere in detail. [35, 38-41].

To ensure the quality of fits, a comparison between the analytical solution of the 1D equation of momentum diffusion and DPD results is presented in Figure 12 for three cases. The first case shows the comparison at constant time step and dissipative parameter, while the shear rate is changing from 0.1 to 2.0 (Figure 12.a). In Figure 12.b, the shear rate is kept constant and the transient velocity after 1000 time steps is compared with the analytical solution, while the dissipative parameter is changing. Finally, at constant shear rate and gamma, the quality of the fitting procedure was examined in different time steps, Figure 12.c. As it can be seen from all the graphs, DPD can produce the correct kinematics in transient shear flow regardless of the level of shear rate and dissipative parameter. 

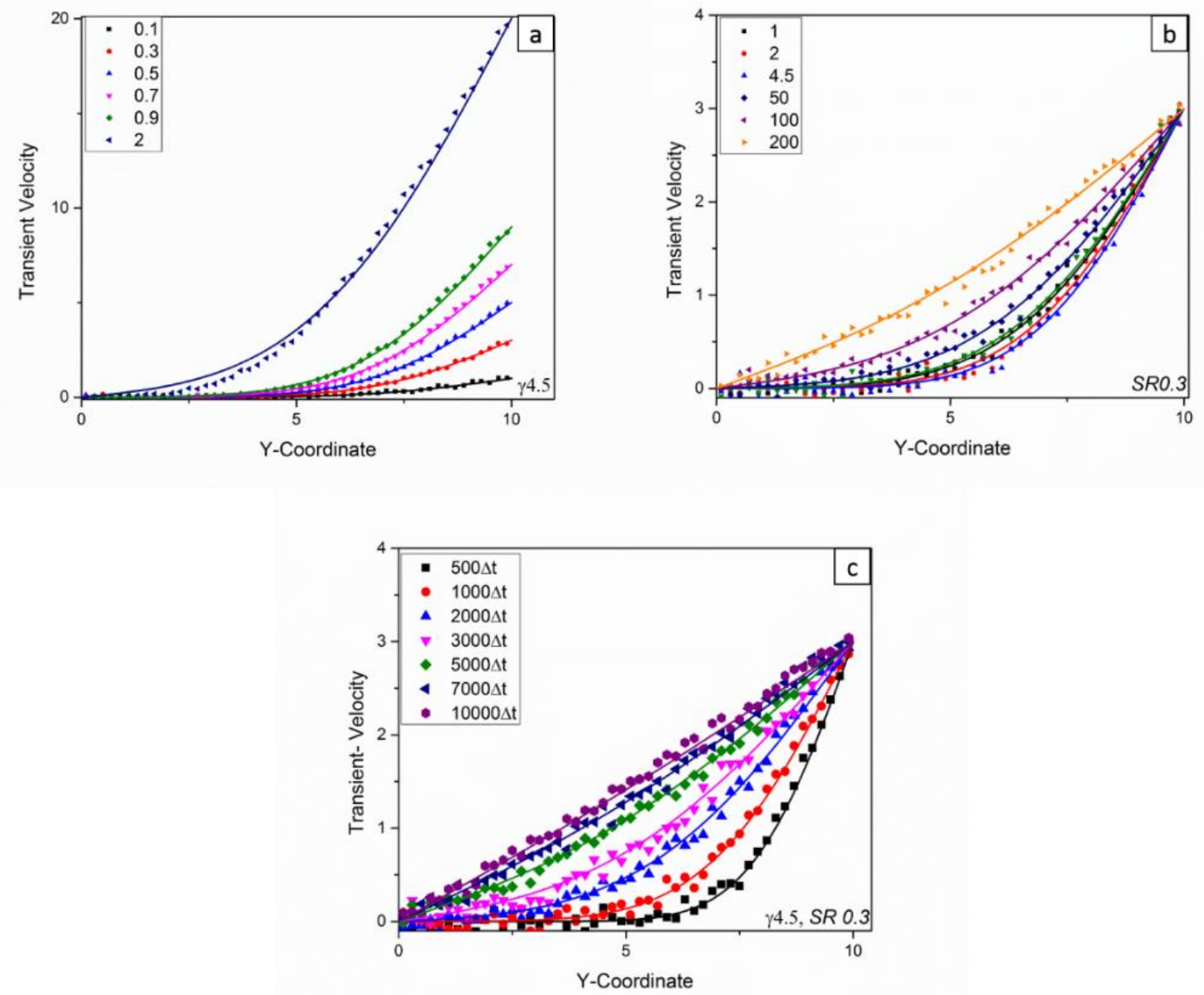

Figure 12: Transient Velocity for (a) different shear rates at constant Gamma after 1000 time steps (b) different values for Gamma at constant shear rate (0.3) after 1000 time steps and (c) different time steps at constant values of shear rate (0.3) and Gamma (4.5)

After assuring that DPD is capable of reproducing the correct kinematics for start-up shear flow, the values for viscosity are calculated from fitting the transient velocity curve obtained after 1000 time steps for unbounded transient shear flow under a constant shear rate of $0.1 \mathrm{~s}^{-1}$. The data are presented in figure 13.a (घ). To ensure that the time step does not influence the obtained values for viscosity, the same procedure was repeated to fit the values of transient velocity after 500 time steps and the obtained values are presented in Figure 13.a (•). Next, The data obtained from PPF method, Table 3, are shown in the 
same graph and as it can been seen both transient start-up shear flow and Poiseuille flow predict the same values and trend for viscosity $(\eta)$ as a function of dissipative parameter $(\gamma)$.

For bounded flows, transient start-up shear flows can be limited to low values of shear-rates. The reason is related to selecting a proper potential between the wall particles and the solvent particles and the fact that at higher shear-rates (boundary velocities) significant slip can occur [18, 24]. However, this problem does not exist in unbounded flows and using proper boundary conditions to impose periodicity in the velocity gradient direction (Eulerian and Lagrangian) will prevent this issue, and higher values of shear rates can be achieved. In this case, the artificial temperature increase will be the limiting factor. For this reason, we calculated the viscosity at different shear rates, as shown in Figure 13.b as a function of the dissipative parameter.
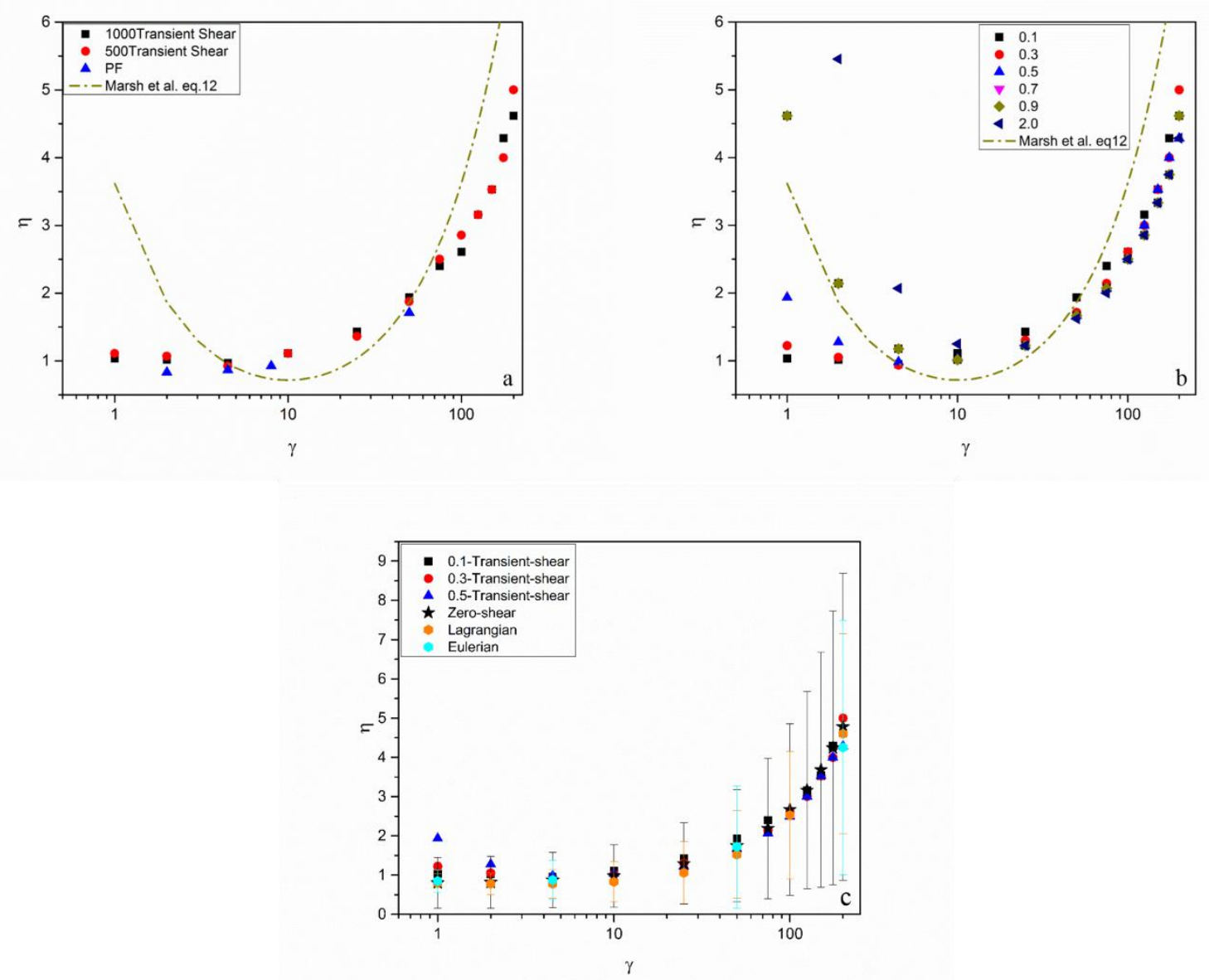

Figure 13: (a) Viscosity values of DPD fluid with different dissipative strengths for transient and Poiseuille flows.(b) viscosity values calculated from transient shear (TS) profile for different shear rates. (c) Comparison between all the methods including the $\operatorname{TSF}(\mathbf{\bullet} \bullet, \mathbf{\Delta})$, Green-Kubo(*), LE-boundary condition( $(\bullet)$ and Lagrangian boundary condition $(\bullet)$ 
As it can be seen from figure 13b, there are three highlights. First, for dissipative parameters higher than 10, while the viscosity increases with increasing gamma, its value does not depend on the applied shear rate. Second, for dissipative parameters less that 10 , the viscosity increases with increasing shear rate that can be attributed to thermal fluctuations in the system. This is due to insufficient dissipative strength to stabilize the temperature (thermostat effect), which is solely a numerical artifact and should not be mistaken by other rheological behavior, e.g. shear-thickening. Thus, precautions are advised when using DPD with $\gamma \leq 10$ to model complex systems, e.g. suspensions in which characteristic shear-thickening is existent. Third, at higher values of dissipative parameter, viscosity values follow qualitatively the Marsh equation. Although Marsh model limits to the systems with no conservative force, it could be inferred that in the case of high dissipative parameter the transport properties are not affected by the presence of the conservative interactions.

Finally, all the results from stress calculation (Eulerian and Lagrangian), zero-shear calculation and transient flow are presented at fig. 13.c. At it can be seen all the results at dissipative parameter above 4.5 falls on the same graph. While the fitting procedure shows the least standard deviation in the stress calculation methods the order is $\left(\sigma_{\text {Langrangian }}<\sigma_{\text {Zero-shear }}<\sigma_{\text {Eulerian }}\right)$ where $\sigma$ is the standard deviation for all the methods. Standard deviations for the fitting procedures are less than 5\%. It could be advised for simple systems (simple rheological materials) and when there is no secondary flows such as migration of internal components such as polymer chains or suspension in PF, fitting procedures should be the primary method used for the viscosity measurement. However, in complex systems our results indicate the Eulerian approach cannot be used below a shear rate of 0.01 and it suffered from high noise to signal ratio.

\section{Conclusions:}

Since DPD is a relatively recent simulation method and the literature on DPD simulations out of equilibrium is rather scattered, a guideline for viscosity measurement using DPD is non-existent. Furthermore, the need for correct boundary conditions and a wider range of shear rates accessible to DPD simulations cannot be neglected. Thus, in this work we performed a series of systematic studies on different methods of viscosity measurements and, more importantly, introduced a boundary condition scheme that successfully broadens the shear rates accessible to DPD simulations to lower shear rates without compromising the computational costs.

In this paper, a comprehensive study was conducted to compare different techniques to measure the viscosity by Dissipative Particle Dynamics, and using two methods. First, using the pressure tensor 
and the microscopic definition of stress in the system; second, using the velocity profile and numerical fitting methods. We also presented a modification to the Lees-Edward (LE) boundary condition, which was originally proposed for MD simulations but is broadly used in DPD. This method superimposes the shear at each time step, while the calculation of the stress is carried out for flow and the material separately. This method will increase the accessible window of low shear rates and produce data with lower noise to signal ratios at higher shear rates when compared to the standard LE boundary condition. We verified the feasibility of the method by observing the velocity profiles at different shear rates and strengths of the dissipative force. We showed that the temperature instabilities and artifacts at high shear rates are functions of the strength of the dissipative force and the fact that using smaller time steps cannot alleviate the problem. To understand the origin of this problem, the velocity distribution at each shear rate for different values of the dissipative parameter was analyzed, and we concluded that higher values of the dissipative parameter control the dissemination of the thermal instabilities in the system better. This fact reveals one of the shortcomings in the DPD scheme, the fact that the dissipative force controls the dynamics of the system while it is also part of the thermostat. To decouple the thermostatting role and solely study the dynamics of the system, we used a stress autocorrelation function to measure the zeroshear viscosity at different temperature and dissipative parameter. The main outcomes of this part are:

- The Groot-Warren scaling law for compressibility out of equilibrium will lead to erroneous results at low dissipative parameter and is due to the diffusion that is resembling gas like behavior.

- We found a scaling relation to determine the critical shear rate at each dissipative parameters below which thermal instabilities can be disregarded, although, there is a need for an auxiliary thermostat for DPD to decouple the temperature control and the dynamics from each other. This point will be addressed in a future study.

- Transient start-up shear flow yields results with the same accuracy of the PPF proposed by Backer et al. and it does not have the problems using PPF such as particle migration and gradient of shear rates.

The second part of this study was dedicated to numerical fitting models mainly periodic Poiseuille flow and transient start-up shear flow. By assuming Newtonian behavior in our system and solving the Navier-Stokes equation followed by velocity fitting procedure, we obtained the viscosity at different values of the dissipative parameter. Both methods provided stable and reliable measurement for viscosity values and are in quantitative agreement with the viscosity measurements through the stress tensor. The measured values for viscosity is in quantitative agreement with the Marsh equation at high values of dissipative parameter that presence of the conservative force does not affect the diffusion of the 
particle. However, we should emphasize that the main assumption of Newtonian behavior is not true in the case of polymer solutions and melts or colloidal suspensions and using microscopic definition of stress cannot be avoided and the lower accessible shear rate can be extended by Lagrangian approach.

\section{Acknowledgment}

A.B acknowledges the NSF Center for Layered Polymeric Systems (CLiPS) for financial support (STC-DMR 0423914). S.J acknowledges NSF funding through Grant No. CMMI/1068960. This work was supported in part by an allocation of computing time from Ohio Supercomputer Center (OSC) and High Power Computing Center (HPCC) of Case Western Reserve University.

\section{REFERENCES:}

1. Hoogerbrugge, P.J. and J.M.V.A. Koelman, Simulating Microscopic Hydrodynamic Phenomena with Dissipative Particle Dynamics. Europhysics Letters, 1992. 19(3): p. 155-160.

2. Groot, R.D. and P.B. Warren, Dissipative particle dynamics: Bridging the gap between atomistic and mesoscopic simulation. J. Chem. Phys., 1997. 107(11): p. 4423-4435.

3. Espanol, P. and P. Warren, Statistical Mechanics of Dissipative Particle Dynamics. Europhysics Letters, 1995. 30(4): p. 191-196.

4. Pivkin, I.V. and G.E. Karniadakis, A new method to impose no-slip boundary conditions in dissipative particle dynamics. J. Comput. Phys., 2005. 207(1): p. 114-128.

5. Pivkin, I.V. and G.E. Karniadakis, Controlling Density Fluctuations in Wall-Bounded Dissipative Particle Dynamics Systems. Physical Review Letters, 2006. 96(20): p. 206001.

6. Backer, J.A., C.P. Lowe, H.C.J. Hoefsloot, and P.D. Iedema, Poiseuille flow to measure the viscosity of particle model fluids. The Journal of Chemical Physics, 2005. 122(15): p. 154503.

7. Chatterjee, A., Modification to Lees-Edwards periodic boundary condition for dissipative particle dynamics simulation with high dissipation rates. Molecular Simulation, 2007. 33(15): p. 1233-1236.

8. Whittle, M. and K.P. Travis, Dynamic Simulations of Colloids by Core-Modified Dissipative Particle Dynamics. Journal of Chemical Physics, 2010. 132: p. 124906(1)-124906(16).

9. Khani, S., M. Yamanoi, and J. Maia, The Lowe-Andersen thermostat as an alternative to the dissipative particle dynamics in the mesoscopic simulation of entangled polymers. Journal of Chemical Physics, 2013. 138(17): p. 174903(1-10).

10. Marsh, C.A., G. Backx, and M.H. Ernst, Fokker-Planck-Boltzmann equation for dissipative particle dynamics. EPL (Europhysics Letters), 1997. 38(6): p. 411.

11. Jamali, S., A. Boromand, and J. Maia, Dissipative Particle Dynamics simulation of colloidal suspensions. Bulletin of the American Physical Society, 2014. 
12. Chatterjee, A. and L.-M. Wu, Predicting rheology of suspensions of spherical and non-spherical particles using dissipative particle dynamics (DPD): methodology and experimental validation. Molecular Simulation, 2008. 34(3): p. 243-250.

13. Padding, J.T. and A.A. Louis, Hydrodynamic interactions and Brownian forces in colloidal suspensions: Coarse-graining over time and length scales. Physical Reviews, 2006. 74: p. 031402.

14. Martys, N.S., Study of a dissipative particle dynamics based approach for modeling suspensions. Journal of Rheology (1978-present), 2005. 49(2): p. 401-424.

15. Jamali, S., M. Yamanoi, and J. Maia, Bridging the gap between microstructure and macroscopic behavior of monodisperse and bimodal colloidal suspensions. Soft Matter, 2013. 9(5): p. 15061515 .

16. Yamanoi, M., O. Pozo, and J. Maia, Linear and non-linear dynamics of entangled linear polymer melts by modified tunable coarse-grained level Dissipative Particle Dynamics. Journal of Chemical Physics, 2011. 135: p. 044904(1-9).

17. Pan, D., N. Phan-Thien, and B.C. Khoo, Dissipative particle dynamics simulation of droplet suspension in shear flow at low Capillary number. Journal of Non-Newtonian Fluid Mechanics, 2014. 212(0): p. 63-72.

18. Pana, D., N. Phan-Thiena, N. Mai-Duya, and B.C. Khoo, Numerical investigations on the compressibility of a DPD fluid. Journal of Computational Physics 2013. 242 p. 196-210.

19. Zhao, T., X. Wang, L. Jiang, and R.G. Larson, Dissipative particle dynamics simulation of dilute polymer solutions - Inertial effects and hydrodynamic interactions. Journal of Rheology (1978present), 2014. 58(4): p. 1039-1058.

20. Clark, A.T., M. Lal, J.N. Ruddock, and P.B. Warren, Mesoscopic Simulation of Drops in Gravitational and Shear Fields. Langmuir, 2000. 16(15): p. 6342-6350.

21. Kubo, R., Statistical-mechanical theory of irreversible processes. I. General theory and simple applications to magnetic and conduction problems. Journal of the Physical Societ of Japan, 1957. 12(6): p. 570-586.

22. Ghoufi, A., J. Emile, and P. Malfreyt, Recent advances in Many Body Dissipative Particles Dynamics simulations of liquid-vapor interfaces. The European Physical Journal E, 2013. 36(1): p. 1-12.

23. Lees, A.W. and S.F. Edwards, The computer study of transport processes under extreme conditions. Journal of Physics C: Solid State Physics, 1972. 5: p. 1921-1929.

24. Fedosov, D.A., I.V. Pivkin, and G.E. Karniadakis, Velocity limit in DPD simulations of wallbounded flows. Journal of Computational Physics, 2008. 227(4): p. 2540-2559.

25. Fedosov, D.A., G.E. Karniadakis, and B. Caswell, Steady shear rheometry of dissipative particle dynamics models of polymer fluids in reverse Poiseuille flow. The Journal of Chemical Physics, 2010. 132(14): p. 144103.

26. Boek, E.S., P.V. Coveney, and H.N.W. Lekkerkerker, Computer simulation of rheological phenomena in dense colloidal suspensions with dissipative particle dynamics. Journal of Physics: Condensed Matter, 1996. 8(47): p. 9509.

27. Boek, E.S., P.V. Coveney, H.N.W. Lekkerkerker, and P. van der Schoot, Simulating the rheology of dense colloidal suspensions using dissipative particle dynamics. Physical Review E, 1997. 55(3): p. 3124-3133. 
28. Chen, S., N. Phan-Thien, B.C. Khoo, and X.J. Fan, Flow around spheres by dissipative particle dynamics. Physics of Fluids (1994-present), 2006. 18(10): p. 103605.

29. Ranjith, S.K., B.S.V. Patnaik, and S. Vedantam, No-slip boundary condition in finite-size dissipative particle dynamics. Journal of Computational Physics, 2013. 232(1): p. 174-188.

30. Marsh, C.A., G. Backx, and M.H. Ernst, Static and dynamic properties of dissipative particle dynamics. Physical Review E, 1997. 56(2): p. 1676-1691.

31. Visser, D.C., H.C.J. Hoefsloot, and P.D. Iedema, Modelling multi-viscosity systems with dissipative particle dynamics. Journal of Computational Physics, 2006. 214: p. 491-504.

32. Revenga, M., I. Zúñiga, P. Español, and I. Pagonabarraga, Boundary Models in DPD. International Journal of Modern Physics C, 1998. 09(08): p. 1319-1328.

33. Revenga, M., I. Zuniga, and P. Espanol, Boundary conditions in dissipative particle dynamics. Comput Phys Commun, 1999. 121: p. 309-311.

34. Visser, D.C., H.C.J. Hoefsloot, and P.D. Iedema, Comprehensive boundary method for solid walls in dissipative particle dynamics. J. Comput. Phys., 2005. 205(2): p. 626-639.

35. Willemsen, S., H. Hoefsloot, and P. Iedema, No-slip boundary condition in dissipative particle dynamics. Int J Mod Phys, 2000. 11: p. 881-890.

36. Pagonabarraga, I., M.H.J. Hagen, and D. Frenkel, Self-consistent dissipative particle dynamics algorithm. EPL (Europhysics Letters), 1998. 42(4): p. 377.

37. Masters, A.J. and P.B. Warren, Kinetic theory for dissipative particle dynamics: The importance of collisions. EPL (Europhysics Letters), 1999. 48(1): p. 1.

38. Haber, S., N. Filipovic, M. Kojic, and A. Tsuda, Dissipative particle dynamics simulation of flow generated by two rotating concentric cylinders: Boundary conditions. Physical Review E, 2006. 74(4): p. 046701.

39. Filipovic, N., S. Haber, M. Kojic, and A. Tsuda, Dissipative particle dynamics simulation of flow generated by two rotating concentric cylinders: II. Lateral dissipative and random forces. Journal of Physics D: Applied Physics, 2008. 41(3): p. 035504.

40. Lei, H., D.A. Fedosov, and G.E. Karniadakis, Time-dependent and outflow boundary conditions for Dissipative Particle Dynamics. Journal of Computational Physics, 2011. 230(10): p. 37653779 .

41. Wagner, A.J. and I. Pagonabarraga, Lees-Edwards Boundary Conditions for Lattice Boltzmann. Journal of Statistical Physics, 2002. 107(1-2): p. 521-537. 